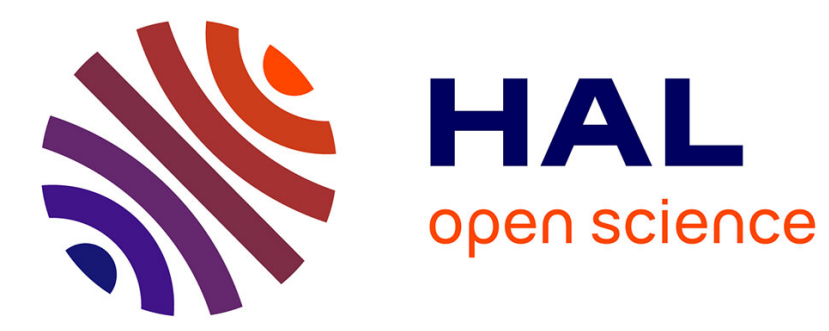

\title{
LOW-DOSE SYNCHROTRON NANO-CT VIA COMPRESSED SENSING
}

\author{
Juan F P J Abascal, C Goubet, Françoise Peyrin, M. Langer
}

\section{To cite this version:}

Juan F P J Abascal, C Goubet, Françoise Peyrin, M. Langer. LOW-DOSE SYNCHROTRON NANOCT VIA COMPRESSED SENSING. The International Symposium on Biomedical Imaging (ISBI'18), Apr 2018, Washington DC, United States. hal-01774857

\section{HAL Id: hal-01774857 https://hal.science/hal-01774857}

Submitted on 24 Apr 2018

HAL is a multi-disciplinary open access archive for the deposit and dissemination of scientific research documents, whether they are published or not. The documents may come from teaching and research institutions in France or abroad, or from public or private research centers.
L'archive ouverte pluridisciplinaire HAL, est destinée au dépôt et à la diffusion de documents scientifiques de niveau recherche, publiés ou non, émanant des établissements d'enseignement et de recherche français ou étrangers, des laboratoires publics ou privés. 


\title{
LOW-DOSE SYNCHROTRON NANO-CT VIA COMPRESSED SENSING
}

\author{
C. Goubet, M. Langer, F. Peyrin, J. F. P. J. Abascal \\ Univ Lyon, INSA-Lyon, Universit Claude Bernard Lyon 1, UJM-Saint Etienne, CNRS, \\ Inserm, CREATIS UMR 5220, U1206, Lyon, France
}

\begin{abstract}
Synchrotron phase nano-CT is a very useful technique for studying bone diseases, which requires investigating bone at the cellular level. Nevertheless, imaging biological tissue at this resolution is challenging due to the very high radiation dose. Compressed sensing provides a framework that permits to reconstruct an image from a limited amount of data. The most promising way to reduce radiation exposure in X-ray $\mathrm{CT}$ is to reduce the number of projections. The aim of this study is to assess the use of compressed sensing to reduce dose for synchrotron phase nano-CT for bone applications. In this paper, we address the tomographic reconstruction step by posing a total variation problem and solving it with the Split Bregman formulation. To assess the proposed method we created different low-dose imaging scenarios by reducing the number of projections, and tested them on several bone samples. The proposed method allowed accurate reconstruction using $1 / 4$ th of the projections, preserving bone features, details, and a high signal to noise ratio.
\end{abstract}

Index Terms - Synchrotron CT, low-dose, compressed sensing, split Bregman

\section{INTRODUCTION}

$\mathrm{X}$-ray CT imaging is a technique of choice to investigate bone in diseases such as osteoporosis. Bone has a sophisticated hierarchical organization, from the organ scale to the nano scale and its strength depends on features at all scales [1,2]. The feasibility of X-ray techniques to analyze bone tissue at the cellular scale has been demonstrated by using ptychography [3], synchrotron micro-CT [4] and phase nano-CT [5]. Nevertheless, imaging biological tissue in $3 \mathrm{D}$ at the nano scale remains very challenging. For instance, bone imaging at a resolution of $60 \mathrm{~nm}$ [5] required acquiring a very large number of projections and long acquisition times (1.9 hours to acquire 2999 projections at four focus-to-sample distances),

\footnotetext{
This project has received funding from the European Union's Horizon 2020 research and innovation programme under the Marie Sklodowska-Curie grant agreement $\mathrm{N}^{\circ}$ 701915. It was also performed within the framework of the LabEx PRIMES (ANR-11-LABX-0063) of University de Lyon. We also acknowledge the support of the ANR project SALTO (ANR-17-CE19-0011$01)$.
}

which translated in a dose equivalent to $8 \cdot 10^{7} \mathrm{~Gy}$. Exposing the sample to a large radiation dose during multiple hours can affect the sample creating motion artefacts and compromising resolution and image quality. In addition, access to synchrotron radiation is limited. Facing these constraints in dose and acquisition time requires new strategies and algorithms that allow for low-dose nano CT.

Compressed sensing (CS) provides a framework that permits to reconstruct an image from a limited amount of data. The most promising way to reduce radiation exposure in $\mathrm{x}-$ ray $\mathrm{CT}$ is to reduce the number of projections acquired $[6$, $7,8]$. CS has been used to reduce the number of projections in micro-CT $[9,10,11]$, but only few studies have addressed synchrotron nano-CT $[12,13]$.

The goal of this study is to assess the use of compressed sensing to reduce dose in synchrotron phase nano CT for bone applications. In this paper, we will only address the tomographic reconstruction step. To this aim we propose a total variation problem and solve it using the Split Bregman formulation, which is an efficient formulation for solving L1based problems $[14,15]$. Similar proximal methods and splitting algorithms have been proposed to solve large scale problems due to their scalability potential $[16,17]$. To assess the proposed method for bone imaging, we created different lowdose imaging scenarios by reducing the number of projections and tested it on several bone samples.

\section{MATERIAL AND METHODS}

\subsection{Compressed sensing formulation}

CS ensures accurate image reconstruction from undersampled data under certain conditions [6]. If $p$ represents data corresponding to a low number of projections, $R$ the forward operator equivalent to a slice-by-slice 2D-Radon transform (for parallel geometry), and $f$ the unknown image that is sparse under a known transformation $\Psi$, then $f$ can be recovered by solving the following convex problem:

$$
\min _{f}\|\Psi f\|_{1} \quad \text { such that } \quad\|R f-p\|_{2}^{2} \leq \sigma^{2},
$$

where $\sigma^{2}$ accounts for noisy data. In this work we consider the $2 \mathrm{D}$ gradient transform $\Psi=\nabla$ that leads to total variation 
functional which isotropic formulation is given by $\|\nabla f\|_{1}=$ $\sqrt{\left(\nabla_{x} f\right)^{2}+\left(\nabla_{y} f\right)^{2}}$.

To solve the problem (1) we use the Split Bregman formulation, which efficiently handles L1-based constrained problems $[14,15,18]$. The Split Bregman formulation allows to split L1-norm and L2-norm terms in such a way that they can both be solved analytically in two separate steps. To allow for splitting, we include new variables, $x$ and $y$, and formulate a new problem that is equivalent to (1):

$$
\begin{aligned}
& \left(f^{k+1}, x^{k+1}, y^{k+1}\right)=\arg \min _{f, x, y}\|(x, y)\|_{1} \\
& \quad+\frac{\lambda}{2}\left\|x-\nabla_{x} f-b_{x}\right\|_{2}^{2}+\frac{\lambda}{2}\left\|y-\nabla_{y} f-b_{y}\right\|_{2}^{2} \\
& \quad+\frac{\mu}{2}\left\|R f-p^{k}\right\|_{2}^{2}, \\
& p^{k+1}=p^{k}+p-R f^{k+1}, \\
& b_{x}^{k+1}=b_{x}^{k}+\nabla_{x} f^{k+1}-x^{k+1}, \\
& b_{y}^{k+1}=b_{y}^{k}+\nabla_{y} f^{k+1}-y^{k+1} .
\end{aligned}
$$

Note that now L2 and L1-terms are independent of each other. The L2 part leads to a linear problem, which is solved analytically, and L1-parts are given by shrinkage formulae. For a detail solution we refer to $[15,8]$. The resulting reconstruction algorithm will be referred as TV-SB.

\subsection{Data and image analysis}

Data were acquired at the European Synchrotron Radiation Facility (ESRF) on beamline ID16A [19]. Sampled corresponded to bone acquired at different propagation distances. A phase retrieval step was applied to each data set of four projections to get a phase map. The set of phase maps retrieved at each projection angle was then reconstructed by filtered back projection (FBP) to get the 3D phase image of size $2048^{3}$ with a voxel size of $120 \mathrm{~nm}$ [20].

The original image had a size of $2048^{3}$ and occupied 60 GB. In order to assess the algorithm in a wide range of scenarios, we created smaller images by extracting three VOIs from the volume reconstructed using fully-sampled data. To evaluate the method of relevant structures of interest in bone at the cellular scale, we selected features representative of the osteo-canalicular system which plays a major role in bone physiology. The first target represents an osteocyte lacuna, the osteocyte being the most abundant cells in bone tissue, the second one represents an osteocyte lacunae including calcium depositium, and the third one targets canaliculi which are the the small channels connecting the osteocyte lacunae (target 1 is displayed in figure 1 and targets 2 and 3 are displayed in figure 2). For each of these VOIs, the projections were simulated numerically.

Low-dose scenarios were created by reducing the number of projections by $1 / 2,1 / 4,1 / 7$ and $1 / 10$. An acquisition was considered fully projected when the number of projections generated was equal to $\pi / 2$ times the image size.

\begin{tabular}{|l||cccc|}
\hline number of projections & $\mathbf{1 / 2}$ & $\mathbf{1 / 4}$ & $\mathbf{1 / 7}$ & $\mathbf{1 / 1 0}$ \\
\hline \hline RMSE (FBP) & $6 \%$ & $7 \%$ & $9 \%$ & $11 \%$ \\
PSNR (FBP) & 68 & 67 & 64 & 62 \\
SAM (FBP) & 5.7 & 8.4 & 14.2 & 18 \\
\hline RMSE (TV-SB) & $1 \%$ & $1 \%$ & $2 \%$ & $2 \%$ \\
PSNR (TV-SB) & 83 & 82 & 79 & 77 \\
SAM (TV-SB) & 1.4 & 1.7 & 2 & 2.5 \\
\hline
\end{tabular}

Table 1. Results using FBP and TV-SB algorithms for $1 / 2$, $1 / 4,1 / 7$, and $1 / 10$ of the total number of projections.

\subsection{Comparison of methods and assessment of image quality}

FBP and TV-SB algorithms were evaluated in terms of several metrics. We used mean squared error (MSE), peak signal to noise ratio (PSNR) and streak artefact measure (SAM) [8]. In addition, reconstructed images were assessed by visual inspection to evaluate the preservation of edges and bone features. The edge preservation was displayed by using canny edge detection. RMSE is given by $\|f-\hat{f}\| /\|\hat{f}\|$, where $\hat{f}$ is the target image, and PSNR as $10 \log _{10}\left(L^{2} / M S E\right)$, where $L$ is the range of the values of the image pixels. The SAM is defined as $T V(f-\hat{f})=\|\nabla(f-\hat{f})\|_{1}$.

We assessed TV-SB and FBP in all scenarios for target 1. Then, we selected the achievable dose reduction and evaluated methods on the other two targets 2 and 3 .

\section{RESULTS}

The different metrics for the target 1 are displayed in Table 1. TV-SB presented a RMSE of $2 \%$ for $1 / 10$ th of the projections while FBP led to a RMSE of $6 \%$ already for one half of the projections and to $11 \%$ for $1 / 10$ th of the projections. For PSNR, good image quality was considered when values were above $70 \mathrm{~dB}$. While TV-SB had PSNR above 70 for all scenarios, FBP presented lower values in all cases. SAM is the most interesting metric as it measures aliasing artefacts and fake edges. TV-SB led to low values of SAM across scenarios. On the contrary, FBP led to a value of SAM larger than 5 for half of the projections and to a three-fold increase for $1 / 10$ th of the projections.

Visual inspection corroborates these results. Figure 1 shows reconstructed images by using FBP and TV-SB and their corresponding edges for the different scenarios for target 1 . For half of the projections, FBP presented noise and artefacts and lost edges with the lowest contrast. TV-SB preserved the most relevant details up to $1 / 4$ th of the projections and retained edges with largest contrast for up to $1 / 10$ th of the projections.

Table 2 and Figure 2 show results for reconstructions by FBP and TV-SB for 1/4th of the projections for all targets. As in target 1, TV-SB retains image quality and bone details 


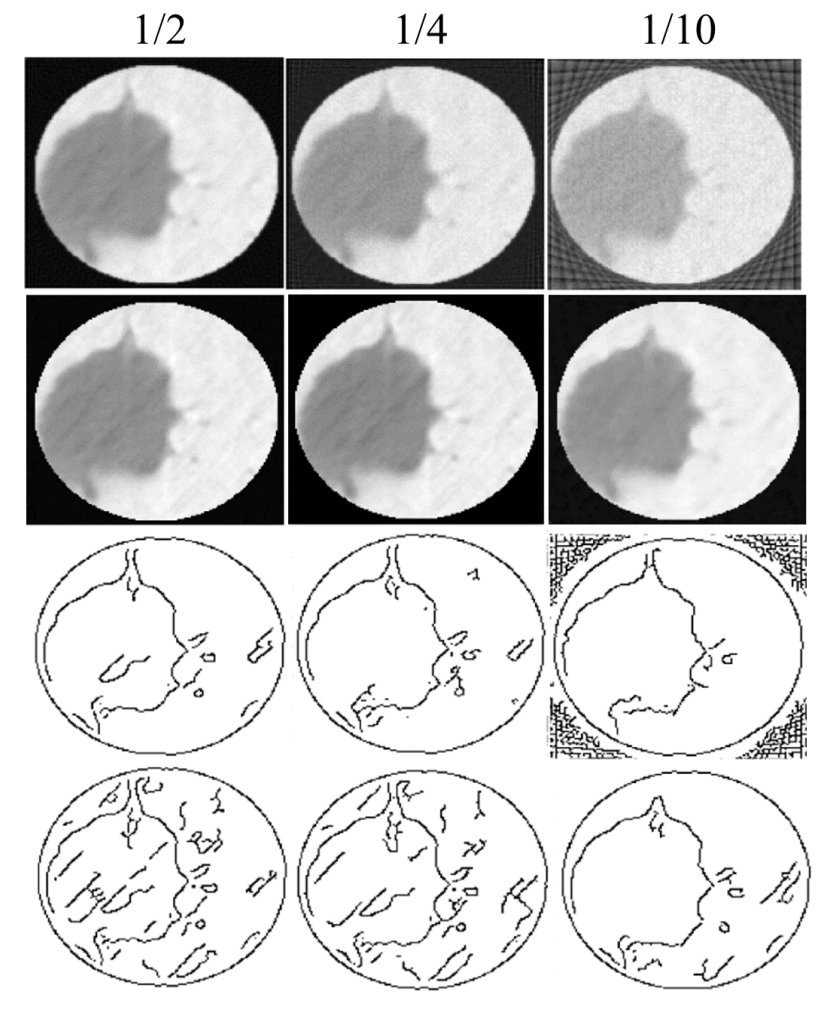

Fig. 1. First and second rows: Reconstructed images using FBP and TV-SB, respectively, for data with projections reduced by $1 / 2,1 / 4$, and $1 / 10$. Third and fourth rows: Edges corresponding to previous images. We consider here target 1 .

\begin{tabular}{|l||ccc|}
\hline & Target 1 & Target 2 & Target 3 \\
\hline \hline RMSE & $1 \%$ & $1 \%$ & $1 \%$ \\
PSNR & 83 & 80 & 82 \\
SAM & 1.4 & 2 & 1.6 \\
\hline
\end{tabular}

Table 2. Results using TV-SB for $1 / 4$ th of the total number of projections and for the three targets.

for these samples.

\section{DISCUSSION}

We proposed a TV-SB algorithm for low dose synchrotron phase nano $\mathrm{CT}$ and validated it on bone data. The results show that TV-SB allows accurate reconstruction using up to $1 / 4$ th of the projections and that higher contrasts can be preserved with $1 / 10$ th of the projections. On the contrary, traditional FBP reconstruction presented noise, blurred edges and details already when using half of the projections.

The total amount of dose that can be reduced with the proposed method depends on the criterion selected for image quality. For bone imaging, post-processing requires bone details and low contrast details to be preserved. Here, we found

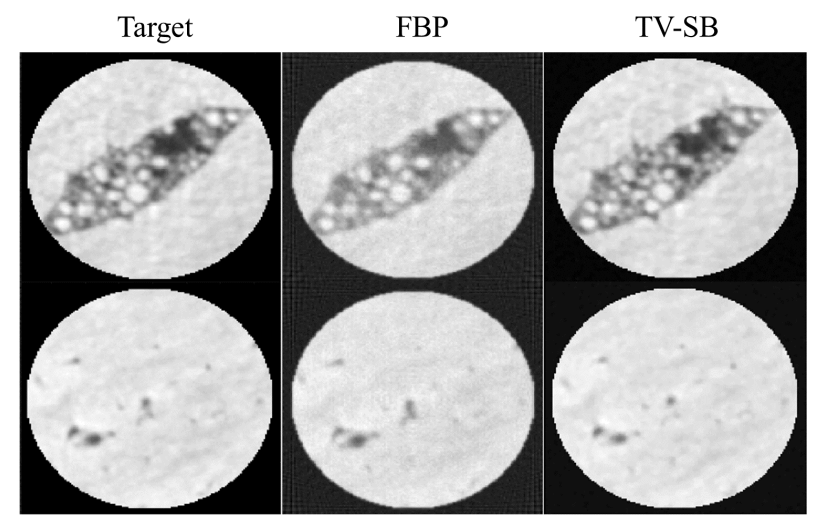

Fig. 2. Reference image and reconstructed images with FBP and TV-SB for $1 / 4$ th of the projections for targets 2 and 3.

that the compressed method could allow a four-fold reduction in the number of projections. This could translate to a fourfold reduction in dose and a significant decrease in acquisition time, which is crucial given the limited access to synchrotron beam time. In addition, decreasing acquisition time has the potential to reduce motion artefacts, which limits resolution and image quality.

In this work we investigated low-dose protocols using compressed sensing. However, this work is subject to few limitations. The methods were assessed on small numerical phantoms since evaluation of the real data set (60 GB) was unfeasible with the current version of the algorithm, due to extended computation times. Future work will address implementation of the proposed algorithm on a cluster, exploiting efficient projection and retroprojection operators that have been developed for ESRF data [21]. Similar proximal methods and splitting algorithms have been proposed to solve large scale problems because of their scalability potential $[16,17]$. In addition, reducing the number of projections would lead to reduced computation times. In this work we reached a four-fold reduction in the number of projections using TV-SB, but larger reduction could be obtained by using a higher order total variation method [22] or another sparsity promoting functional.

\section{ACKNOWLEDGMENTS}

We thank beam team at ESRF ID16A, Alexandra Pacureanu, and Peter Cloetens from ESRF and Boliang Yu and Cécile Oliver from CREATIS.

\section{REFERENCES}

[1] E. Seeman and P. D. Delmas, "Bone Quality The Material and Structural Basis of Bone Strength and Fragility," N. Engl. J. Med., vol. 354, no. 21, pp. 2250-2261, 2006. 
[2] P. Schneider, M. Meier, R. Wepf, and R. Müller, "Towards quantitative 3D imaging of the osteocyte lacunocanalicular network.” Bone, vol. 47, no. 5, pp. 848-58, 2010.

[3] M. Dierolf, A. Menzel, P. Thibault, P. Schneider, C. M. Kewish, R. Wepf, O. Bunk, and F. Pfeiffer, "Ptychographic X-ray computed tomography at the nanoscale," Nature, vol. 467, no. 7314, pp. 436-439, 2010.

[4] A. Pacureanu, M. Langer, E. Boller, P. Tafforeau, and F. Peyrin, "Nanoscale imaging of the bone cell network with synchrotron X-ray tomography: optimization of acquisition setup." Med. Phys., vol. 39, no. 4, pp. 222938, 2012.

[5] M. Langer, A. Pacureanu, H. Suhonen, Q. Grimal, P. Cloetens, and F. Peyrin, "X-ray phase nanotomography resolves the 3D human bone ultrastructure." PLoS One, vol. 7, no. 8, p. e35691, 2012.

[6] E. J. Candès, J. K. Romberg, and T. Tao, "Stable signal recovery from incomplete and inaccurate measurements," Comm. Pure Appl. Math, vol. 59, no. 8, pp. 1207-1223, 2006.

[7] X. Pan, E. Y. Sidky, and M. Vannier, "Why do commercial CT scanners still employ traditional, filtered backprojection for image reconstruction?" Inverse Probl., vol. 25, no. 12, p. 1230009, 2009.

[8] J. Abascal, M. Abella, E. Marinetto, J. Pascau, and M. Desco, "A novel prior- and motion-based compressed sensing method for small-animal respiratory gated CT," PLoS One, vol. 11, no. 3, 2016.

[9] X. Li and S. Luo, "A compressed sensing-based iterative algorithm for CT reconstruction and its possible application to phase contrast imaging," J. Biomed. Opt., vol. 10, no. 1, p. 73, 2011.

[10] Y. Zhao, E. Brun, P. Coan, Z. Huang, A. Sztrókay, P. C. Diemoz, S. Liebhardt, A. Mittone, S. Gasilov, J. Miao, and A. Bravin, "High-resolution, low-dose phase contrast X-ray tomography for 3D diagnosis of human breast cancers." Proc. Natl. Acad. Sci. U.S.A., vol. 109, no. 45, pp. 18 290-4, 2012.

[11] Y. Liu, J. Nelson, C. Holzner, J. C. Andrews, and P. Pianetta, "Recent advances in synchrotron-based hard xray phase contrast imaging," J. Phys. D Appl. Phys., vol. 46, no. 49, p. 494001, 2013.

[12] P. Villanueva-Perez, F. Arcadu, P. Cloetens, and M. Stampanoni, "Contrast-transfer-function phase retrieval based on compressed sensing," Opt. Lett., vol. 42, no. 6, p. 1133, 2017.
[13] S. A. Melli, K. A. Wahid, P. Babyn, J. Montgomery, E. Snead, A. El-Gayed, M. Pettitt, B. Wolkowski, and M. Wesolowski, "A compressed sensing based reconstruction algorithm for synchrotron source propagationbased X-ray phase contrast computed tomography," Nucl. Instr. Meth. Phys. Res. A, vol. 806, pp. 307-317, 2016.

[14] S. Osher, M. Burger, D. Goldfarb, J. Xu, and W. Yin, "An Iterative Regularization Method for Total VariationBased Image Restoration," Multiscale Modeling \& Simulation, vol. 4, no. 2, pp. 460-489, 2005.

[15] T. Goldstein and S. Osher, "The Split Bregman Method for L1-Regularized Problems," SIAM J. Imaging. Sci., vol. 2, no. 2, pp. 323-343, 2009.

[16] J. Chamorro-Servent, J. F. P. J. Abascal, J. Aguirre, S. Arridge, T. Correia, J. Ripoll, M. Desco, and J. J. Vaquero, "Use of Split Bregman denoising for iterative reconstruction in fluorescence diffuse optical tomography," J. Biomed. Opt., vol. 18, no. 7, p. 076016, 2013.

[17] A. Onose, R. E. Carrillo, A. Repetti, J. D. McEwen, J.P. Thiran, J.-C. Pesquet, Y. Wiaux, and D. K., "Scalable splitting algorithms for big-data interferometric imaging in the SKA era," Mon. Notices Royal Astron. Soc., vol. 462, no. 4, pp. 4314-4335, 2016.

[18] J. F. P.-J. Abascal, J. Chamorro-Servent, J. Aguirre, S. Arridge, T. Correia, J. Ripoll, J. J. Vaquero, and M. Desco, "Fluorescence diffuse optical tomography using the split Bregman method," Med. Phys., vol. 38, no. 11, pp. 6275-6284, 2011.

[19] B. Yu, M. Langer, A. Pacureanu, R. Gauthier, C. Olivier, H. Follet, D. Mitton, P. Cloetens, and F. Peyrin, "Assessment of imaging quality in magnified phase CT of human bone tissue at the nanoscale," in SPIE Developments in Dev in X Ray Tomography, 2017, pp. Paper 10391-20.

[20] M. Langer, P. Cloetens, J.-P. Guigay, and F. Peyrin, "Quantitative comparison of direct phase retrieval algorithms in in-line phase tomography," Med. Phys., vol. 35 , no. 10, pp. 4556-4566, 2008.

[21] A. Mirone, E. Brun, E. Gouillart, P. Tafforeau, and J. Kieffer, "The PyHST2 hybrid distributed code for high speed tomographic reconstruction with iterative reconstruction and a priori knowledge capabilities," Nucl. Instr. Meth. Phys. Res. B, vol. 324, pp. 41-48, 2014.

[22] K. Bredies, K. Kunisch, and T. Pock, "Total Generalized Variation," SIAM J. Imaging. Sci., vol. 3, no. 3, pp. 492526, 2010. 